\title{
Theoretical Investigation of Field-Line Quality in a Driven Spheromak
}

R.H. Cohen, H.L. Berk, B.I. Cohen, T.K. Fowler, A.H. Glasser, E.B. Hooper, L.L LoDestro, E.C. Morse, L.D. Pearlstein, T.D. Rognlien, D.D. Ryutov, C.R. Sovinec, W. Woodruff

This article was submitted to $19^{\text {th }}$ International Atomic Energy Agency Fusion Energy Conference, Lyon, France, October 14-19, 2002

U.S. Department of Energy

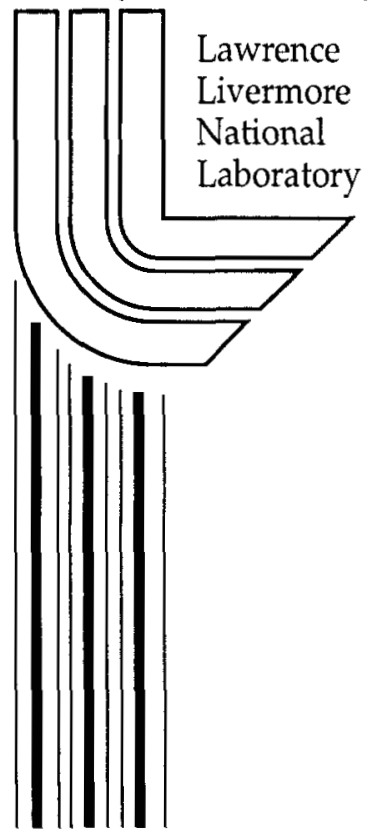

October 7, 2002 


\section{DISCLAIMER}

This document was prepared as an account of work sponsored by an agency of the United States Government. Neither the United States Government nor the University of California nor any of their employees, makes any warranty, express or implied, or assumes any legal liability or responsibility for the accuracy, completeness, or usefulness of any information, apparatus, product, or process disclosed, or represents that its use would not infringe privately owned rights. Reference herein to any specific commercial product, process, or service by trade name, trademark, manufacturer, or otherwise, does not necessarily constitute or imply its endorsement, recommendation, or favoring by the United States Government or the University of California. The views and opinions of authors expressed herein do not necessarily state or reflect those of the United States Government or the University of California, and shall not be used for advertising or product endorsement purposes.

This is a preprint of a paper intended for publication in a journal or proceedings. Since changes may be made before publication, this preprint is made available with the understanding that it will not be cited or reproduced without the permission of the author.

This report has been reproduced directly from the best available copy.

Available electronically at http://www.doc.gov/bridge

Available for a processing fee to U.S. Department of Energy

And its contractors in paper from

U.S. Department of Energy

Office of Scientific and Technical Information

P.O. Box 62

Oak Ridge, TN 37831-0062

Telephone: (865) 576-8401

Facsimile: (865) 576-5728

E-mail: reports@adonis.osti.gov

Available for the sale to the public from

U.S. Department of Commerce

National Technical Information Service

5285 Port Royal Road

Springfield, VA 22161

Telephone: (800) 553-6847

Facsimile: (703) 605-6900

E-mail: orders@ntis.fedworld.gov

Online ordering: http://www.ntis.gov/ordering.htm

OR

Lawrence Livermore National Laboratory

Technical Information Department's Digital Library

http://www.llnl.gov/tid/Library.html 


\title{
Theoretical investigation of field-line quality in a driven spheromak ${ }^{1}$
}

\author{
R.H. Cohen ${ }^{1)}$, H.L. Berk ${ }^{2)}$, B.I. Cohen ${ }^{1)}$, T.K. Fowler ${ }^{3)}$, A.H. Glasser ${ }^{4)}$, \\ E.B. Hooper ${ }^{1)}$,L.L. LoDestro ${ }^{1)}$, E.C. Morse ${ }^{3)}$, L.D. Pearlstein ${ }^{1)}$,T.D. Rognlien ${ }^{1)}$, \\ D.D. Ryutov ${ }^{1)}$, C.R. Sovinec ${ }^{5)}$, S. Woodruff ${ }^{1)}$
}

1) Lawrence Livermore National Laboratory, Livermore CA 94550 USA

2) Institute for Fusion Studies, University of Texas, Austin, TX 78712 USA

3) Department of Nuclear Engineering, University of California, Berkeley, CA 94720 USA

4) Los Alamos National Laboratory, Los Alamos NM 85745 USA

5) University of Wisconsin, Madison WI 53706 USA

e-mail contact of main author: rcohen@llnl.gov

Abstract. Theoretical studies aimed at predicting and diagnosing field-line quality in a spheromak are described. These include nonlinear 3-D MHD simulations, stability studies, analyses of confinement in spheromaks dominated by either open (stochastic) field lines or approximate flux surfaces, and a theory of fast electrons as a probe of field-line length.

\section{Introduction}

Sustainment of a spheromak requires injection of current to balance Ohmic dissipation. This is most commonly done by driving current on the open field lines of the device. It is well known that penetration of this current into the interior of the device requires breaking of magnetic surfaces (which may occur spontaneously due to magnetic fluctuations from, for example, tearing modes), at some cost in terms of energy confinement. It is thus important to determine the length and other statistical properties of the field lines. This paper describes theoretical studies aimed at predicting and diagnosing field-line quality.

\section{NIMROD simulations}

Three-dimensional resistive-MHD simulations have been performed with the NIMROD code for the configuration of the Sustained Spheromak Physics Experiment (SSPX).[1] With caveats about resolution, these simulations semi-quantitatively agree with experiment on many features: magnetic flux amplification, strongly driven (by axisymmetric electrostatic fields) spheromak formation mediated by an $n=1$ current-driven instability (seen in previous simulations [2] and the SPHEX experiment [3]), and spatial dependences of $\lambda=\mu_{0} \mathbf{J} \cdot \mathbf{B} / B^{2}$ and $q$.

The magnetic field lines are typically stochastic and open in simulations approaching a driven steady state due to the presence of the $n=1$ mode and harmonics. Simulations of the formation of the spheromak indicate that the $n=1$ mode can occur for finite or zero resistivity and plasma pressure. (We also simulated axisymmetric spheromak formation in a gun geometry by suppressing all of the toroidal modes except for $n=0$. However, with finite-n toroidal perturbations, there was always significant $n=1$ mode activity during the driven spheromak formation.) With finite pressure, thermal conductivity, and Spitzer temperature-dependent resistivity (for $T_{e} \sim 100 \mathrm{eV}$ giving $\beta_{e} \sim 6 \%$ ), we observed continued buildup of the magnetic energy for pulse times of increasing length (but shorter than the resistive time) and plasmas with pressure profiles that peaked well away from the flux-conserver walls although the field lines were open. In a simulation with an initially cold plasma $\left(T_{e} \sim 5 \mathrm{eV}\right)$, Spitzer resisitivity $\left(\eta \sim 10^{-4}-10^{-5}\right.$ Ohm-m), and anisotropic thermal conductivities $\left(\kappa_{\|}=10^{5} \mathrm{~m}^{2} / \mathrm{s}, \kappa_{\perp}=10^{4} \mathrm{~m}^{2} / \mathrm{s}\right)$, the peak values of the convection/compression, thermal conduction, and Joule heating terms were comparable, suggesting that significant corrections may sometimes be needed to one-dimensional open-field line models such as are discussed in Sec. 7 . The peak $\beta_{e} \sim 0.8 \%, T_{e} \rightarrow 20 \mathrm{eV}$, and the pressure profile was peaked and smooth at $0.2 \mathrm{~ms}$. These results are reasonable for an SSPX plasma during formation with no closed field lines and a large-amplitude $n=1$ mode present

\footnotetext{
${ }^{1}$ Work performed for the U.S. Department of Energy under contracts W7405-ENG-48 at U.C. LLNL, W-7405ENG-36 at U.C. LANL, and DE-FG03-96ER-54346 at U. Texas, and grant FG02-01ER54661 at U. Wisconsin
} 
(although in the experiment $n=1$ mode amplitudes are lower and $T_{e}$ is often higher than in the simulations).
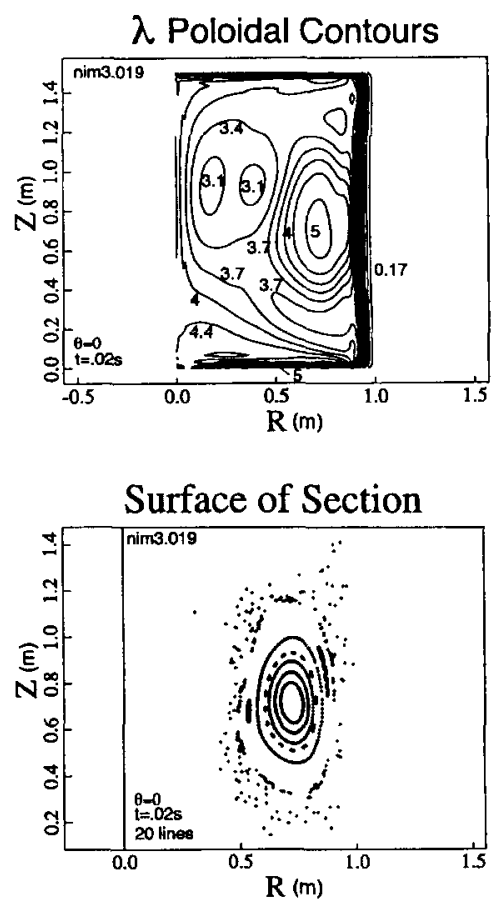

FIG. 1: Emergence of closed field lines and relatively smooth $\lambda$ spatial profiles during decay.

During spheromak formation closed field lines appeared transiently accompanied by current filaments in the simulations, and the poloidal $\lambda$ profiles had substantial gradients. With a steady electrostatic drive and significant $n=1$ mode activity, all field lines were open. However, when the electrostatic drive was crowbarred off, with finite resistivity, the finite- $n$ modes decayed faster than did the toroidally symmetric component; and closed field lines emerged over substantial volumes (Fig. 1). With a pulsed driving electrostatic field, the simulations showed that closed field-line configurations could be produced repetitively in time. The emergence of closed field lines depends sensitively on the relative amplitude of symmetry-breaking perturbations: the magnetic energy in the $n=1$ mode and other finite- $n$ modes must be less than a few percent of the $n=0$ magnetic energy for closed field lines to emerge.

We have postulated that a spheromak with good flux surfaces could be sustained if the open field-line region were subject only to instabilities that would transfer helicity to the spheromak core in a region localized near its edge, and current would be transported to the center by a (possibly intermittently) overlapping chain of high-order, localized islands. To begin to explore this hypothesis, we performed a driven NIMROD simulation which keeps only even toroidal harmonics (and so eliminates coupling to the global $n=1$ mode). This is started from an axisymmetric spheromak equilibrium generated within NIMROD, introducing a small perturbation to the even harmonics, and following the subsequent evolution. For comparison, we repeated the procedure with both even and odd harmoncs. The all-harmonics run, like the build-up runs described above, shows no closed flux surfaces after the harmonics saturate. However, the run with even harmonics has a significant volume of closed flux surfaces and "breathes" (see Fig. 2): the mode amplitudes exhibit quasi-periodic peaking, and the closed-flux-surface volume shrinks and grows over the course of a peaking cycle (a mechanism for replenishing helicity). The transiently stochastic field lines are long, making 60 or more toroidal transits before exiting. We are addressing the outstanding question of whether we can sustain a spheromak configuration with little or no $n=1$ instability.
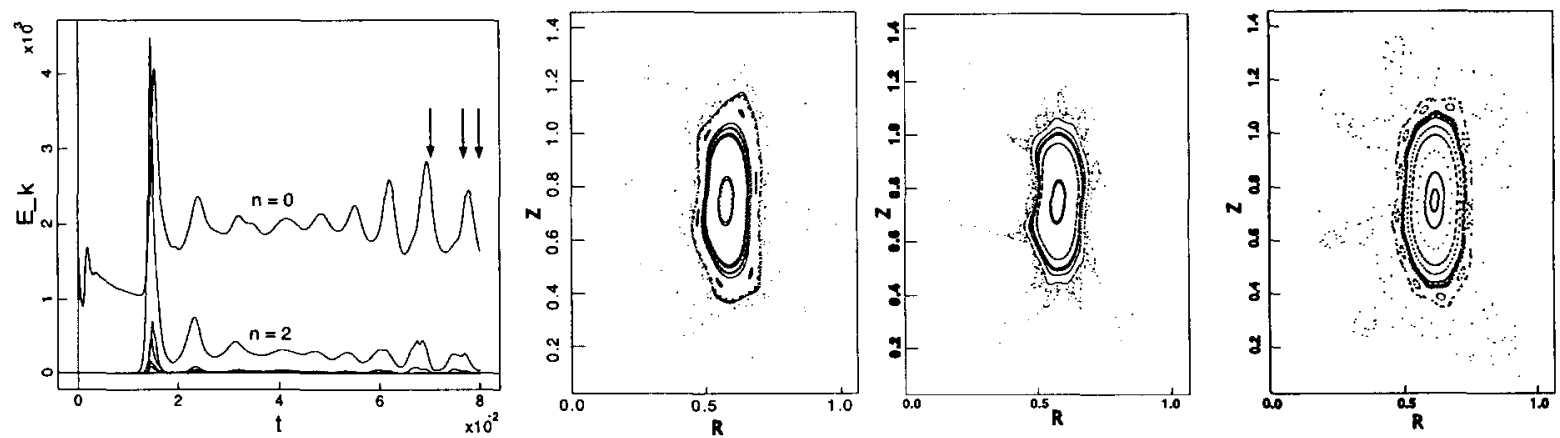

FIG. 2: (a) Kinetic energy per mode versus time. (b) Surfaces of section at three successive times denoted by arrows in (a). 


\section{Galerkin MHD modeling}

As a faster alternative to NIMROD, we have also used a Galerkin MHD model $[4,5]$.In this model, magnetic fields and velocity are represented by a spectral decomposition in terms of basis functions which are eigenfunctions of the curl, $\nabla \times \mathbf{B}_{p}=\lambda_{p} \mathbf{B}_{p}$, such that $\mathbf{B}=\sum_{p} a_{p} \mathbf{B}_{p}$ and $\mathbf{v}=\sum_{p} v_{p} \mathbf{B}_{p}$. The resulting dynamical equations for $\mathbf{B}$ and $\mathbf{v}$ are a set of $2 N$ coupled ordinary differential equations with coupling terms which are bilinear in $a_{p}$ and $v_{p}$. We have deployed this model in two geometries: cylindrical with periodic boundary conditions, and spherical.

Cylindrical modeling has shown magnetic island formation and has revealed parity dependence for the growth of ideal and resistive MHD modes. Resistive growth rates and thresholds correspond with theoretical calculations [6]. In both cylindrical and spherical geometries, driven spheromaks have been modeled by the addition of a source term $S_{p}$ to the MHD Galerkin equations, i. e. $\left(d a_{p} / d t\right)=\ldots+S_{p}$, with $S_{p} \propto V_{\text {gun }}$, where $V_{\text {gun }}$ is the voltage at the Marshall gun electrode. By adding energy into modes with higher helicity $\lambda_{p}$ than the Taylor state, a turbulent mixture of non-axisymmetric states evolves to the lowest-helicity symmetric mode during the decay, in agreement with NIMROD predictions. The spherical Galerkin code, with a screwpinch source and seventy-two field and velocity components in $n=0$ and $n=1$ modes, has been directly compared to NIMROD with a similar drive scenario. Both codes are in agreement for early times as the $n=1$ mode grows at the expense of $n=0$ mode energy, but diverge when $n=2,3,4,5$ mode amplitudes in NIMROD become significant. Future comparisons will include these higher-order modes in the Galerkin code.

\section{MHD stability}

Prediction of field-line quality requires an understanding of the underlying electromagnetic instabilities. By operating SSPX at low density, small-scale fluctuations are observed that appear un-correlated around the machine. In order to identify the instability that caused the fluctuations, we performed a Mercier stability calculation in the CORSICA 11 1/2 D transport code to determine the marginally stable pressure profile for a given set of equilibria (reconstructed from edge field measurements). These profiles were then compared with electron pressure profiles measured with a Thomson Scattering diagnostic. We found that the form of these electron pressure profiles appeared qualitatively similar, and allowed us to compare its peak pressure in the core as a function of time. We found that the peak Mercier-stable pressure tracked the peak measured electron pressure for the duration of the sustainment period $(\sim 500 \mu \mathrm{s})$. This suggests that Mercier instability is limiting the pressure. Moreover, this process would produce fluctuations much like the observed fine-scale magnetic fluctuations.

We examine stability to shift/tilt using the DCON low-mode-number stability package in the CORSICA code. The equilibrium is computed with no current on open field lines (due to a DCON limitation). With the flux conserver as a perfectly conducting wall, the plasma is predicted to be stable. Adding the external currents should effectively bring the wall closer and make the plasma more stable.

\section{Scaling analysis}

We have re-examined the Connor-Taylor[7] scaling analysis of transport in a reversed-field pinch. In this analysis, one seeks transformations of variables that leave the equations (in this case, large-aspect-ratio resistive MHD, with radially localized perturbations) invariant. The number $N$ of such transformations generally rises as simplifications are made to the equations, and the greater $N$, the more completely is prescribed the possible parametric dependence of derived quantities such as transport coefficients. We find that fewer approximations are required to uniquely determine the scaling than is asserted in Ref. [7]. Assuming only that radial localization is better than poloidal (in the notation of [7], $\nabla_{\perp} \sim \sigma \partial / \partial x$, where $\sigma$ is magnetic shear) and resistivity dominates inertia in the induction equation (two of the three conditions in Ref. [7]), we find unique scaling, because there is an additional invariance transformation $(A \rightarrow \lambda A, \Phi \rightarrow \lambda \Phi$, $y \rightarrow \lambda y$, and $\beta^{+} \rightarrow \lambda^{2} \beta^{+}$.) The resultant implied scaling for diffusion due to fluid convection is $D_{0} \sim r B_{\theta} /\left(\rho^{1 / 2} S\right)$, where $S$ is the Lundquist number. This scaling shares the $1 / S$ scaling obtained in Ref. [7], but differs in other dependencies; a posteriori, the approximations made 
are justified in the limit of large poloidal beta. We have also (following Ref. [7]) derived from $D_{0}$ expressions for diffusion due to stochastic field lines. We find other simplifications, with different regimes of applicability, that yield different scalings; in particular, Connor and Taylor's scalings are recovered for approximations justified in the limit $\sigma \gg 1$.

We have also applied the analysis to an expansion of the resistive MHD equations for perturbations radially localized about a Rosenbluth-Bussac force-free spheromak equilibrium [8]. This system has complicated geometrical coefficients, but we nevertheless find that the scaling for all quantities is the same as for the set of RFP approximations explicitly given above. Of course this optimistic scaling can only be expected to apply for radially localized perturbations, which is consistent with the route hypothesized in Sec. 2 for achieving a "good spheromak".

\section{Hyper-resistive modeling}

If the quality of the magnetic surfaces is sufficiently good and magnetic fluctuations are fine-scale, current penetration can be described by a helicity-conserving term (hyper-resistivity) added to Ohm's Law [9]. The resulting surface-averaged flux-diffusion equation now also diffuses current. It is fourth-order and requires an additional boundary condition at the edge of the confinement region. We fix $\mathbf{J}_{\|} / \mathbf{B}$ there and determine the rate of helicity injection. This equation is then solved in conjunction with the free-boundary equilibrium, including current on the open field lines, using the CORSICA code.

We apply our analysis to two SSPX shots. For Shot 7052 at $1.8 \mathrm{~ms}$, when the current profile is at most slowly evolving, we calculate a hyper-resistive steady state with a constant $\Lambda$ value $(0.004 \mathrm{~V}-\mathrm{Wb})$ chosen to yield a best match with magnetic probe data [10]. The calculated rate of helicity transport across the separatrix, $0.62 \mathrm{~V}-\mathrm{Wb}$, is small compared to the helicity supplied by the gun; and at the calculated value of gun flux which passes through the poloidal hole, 0.017 $\mathrm{Wb}$, the effective voltage applied to the confined spheromak is $19 \mathrm{~V}$, much less than the applied voltage $(\sim 350 \mathrm{~V})$. This is consistent with experimental measurements which find that the bulk of the voltage drop is across the sheath and along the open field lines. For Shot 7226, we compare time-dependent simulations with probe data at several times during build-up and sustainment (with $n=1$ dominant). $\Lambda$ is again constant, now chosen to best track the experiment. We find the agreement (rms errors $\sim 2 \%-4 \%$ ) is almost as good as with our usual equilibrium fits, a somewhat surprising result showing that the hyper-resistive model can reasonably well describe current transport even when a (global) $n=1$ mode is dominant.

In the future we plan to use a physics-based $\Lambda$ rather than the empirical choice reported here. There has been considerable progress along this line when the physical drive is tearing modes; the theory has been tested in an RFP configuration. This analysis uses cylindrical $\Delta^{\prime}$ and will be published elsewhere (Berk et al.). When an appropriate tearing mode analysis is available for tight aspect ratios, this theory will be applied to spheromaks.

\section{Classical transport on open field lines}

As noted earlier, our NIMROD simulations often evolve to a steady state in which most or all the field lines are open. The current is driven by the applied static voltage. Following Refs. $[12,11,13]$, we analyze thermal equilibrium with Joule heating balancing electron heat losses along the field lines. We include effects of enthalpy flux and flux limitations near the electrodes. We find that these two effects have only a minor influence on the temperature distribution, which is very flat, except for a sharp drop within a short distance from the electrodes $(<0.1 L$, where $L$ is the connection length). The temperature in the plateau area can be expressed in terms of the voltage $U$ applied to the plasma (i.e., without the sheath potentials) as $T_{0}(\mathrm{eV})=0.39 U(\mathrm{~V})$. The current density is related to $U$ by $j\left(\mathrm{~A} / \mathrm{cm}^{2}\right) \approx 2.9[U(\mathrm{~V})]^{5 / 2} / Z_{\mathrm{eff}} L(\mathrm{~cm})$. However, the aforementioned relationships seem to contradict SSPX experimental results in the following ways: i) Experimentally measured temperature profiles are never flat; ii) The current density is not too sensitive to the applied voltage (contrary to the $j \sim U^{5 / 2}$ prediction); iii) The current density required to sustain the observed temperature for $L<10 \mathrm{~m}$ is much greater than that observed. So, these considerations demonstrate that in the SSPX plasma there must be substantial inductive, convective, or compressive effects (related possibly to fluctuations and often important in 
NIMROD simulations), or the length of field lines at $T \sim 100 \mathrm{eV}$ must be at least $20-30 \mathrm{~m}$.

The experimentally measured current density is a few times larger than the saturation current density $e n v_{t i}$ evaluated for the bulk plasma parameters. We suggest that the higher-thansaturation current is attained via formation of a centimeter-thick layer with a relatively high density of recycled neutral atoms near the cathode; the ionization of these atoms serves as a source of electrons for sustaining a high current density in a bulk plasma, whereas the ions fall back to the cathode. Detailed ID numerical simulations show that, for SSPX parameters, this mechanism can provide the necessary current density.

\section{Fast-electron probe}

The quality of field lines in an operating spheromak can be probed by fast electrons which, on the one hand, do not experience Coulomb scattering and, on the other hand, still have small gyro-radii, so that they closely follow magnetic field lines. Therefore, formation of runaway electrons (detected by their X-ray radiation) can be a sensitive indicator of the structure of the magnetic field lines. We have shown that generation of $\sim 100 \mathrm{keV}$ electrons in SSPX would necessarily indicate the presence of field lines making at least a few thousands of turns. We have also estimated the number of runaway electrons that one can expect to be formed in SSPX under the assumption that the field lines are long enough. This number (for 100-keV electrons) exceeds $10^{-3}$ - more than sufficient for their detection. Our results fit well the existing data on runaway electrons in the CTX spheromak [14].

\section{Conclusion}

We have outlined a number of theoretical/computational tools studies aimed at assessing, understanding, and ultimately improving the quality of field lines in a spheromak, and modeling the transport properties in a spheromak with "good" field lines (approximate flux surfaces and finescale fluctuations). It is evident that large-scale low- $n$ modes, in particular $n=1$, must be kept to a small level - a few percent or less - to achieve a desirable configuraition. Our studies also suggest that such a spheromak should scale well to larger, hotter devices. Further exploration of pulsed scenarios and configurations that reduce the $n=1$ instability are promising directions to pursue.

\section{References}

[1] HILL, D.N., BULMER, R.H., COHEN, B.I., HOOPER, E.B., et al., "Spheromak Formation Studies in SSPX", in Proceedings of the Eighteenth International Conference on Plasma Physics and Controlled Nuclear Fusion Research, Sorrento, Italy, October 4-10, 2000.

[2] SOVINEC, C.R., FINN, J.M., DEL-CASTILlO-NEGRETE, D., Phys. Plasmas 8 (2001) 475.

[3] DUCK, R.C., BROWNING, P.K., CUNNINGHAM, G., GEE, S.J., AL-KARKHY, A., MARTIN, R., RUSBRIDGE, M.G., Plasma Phys. Control. Fusion 39 (1997) 715-736.

[4] CHEN, H., SHAN, X., MONTGOMERY, D., Phys. Rev. A 42 (1990) 6158.

[5] HUA, D.D., FOWLER, T. K., MORSE, E.C., J. Plasma Physics 66 (2001) 275.

[6] GIBSON, R. D., AND WHITEMAN, K. J., Plasma Phys. 10, 1101 (1968).

[7] CONNOR, J.W., TAYLOR, J.B., Phys. Fluids 27 (1984) 2676.

[8] ROSENBUTH, M.N., BUSSAC, M.N. Nucl. Fusion19 (1979) 489.

[9] BOOZER, A.H., J. Plasma Physics 35 (1986) 133.

[10] HOOPER, E.B., PEARLSTEIN, L.D., Plasma Physics Reports 28 (2002) 765.

[11] HOOPER, E.B., COHEN, AND R.H., RYUTOV, D.D., J. Nucl. Materials 278 (2000) 104.

[12] COHEN, R.H. , HOOPER, E.B., RYUTOV., D.D., in: "Theoretical issues in spheromak research," LLNL report UCRL-ID-127002, April 1997.

[13] MOSES, R.W., GERWIN, R.A., SCHOENBERG, K.F., Physics of Plasmas 8 (2001) 4839.

[14] CHRIEN, R.E., FERNANDEZ, J.C., HENINS, I., MAYO, R.M., WYSOCKI, F.J., Nucl. Fus., 31 (1991) 1390. 